\title{
Cervical vertebral maturation (CVM) stage as a supplementary indicator for the assessment of peak height velocity (PHV) in adolescent idiopathic scoliosis (AIS)
}

\author{
Yuancheng Zhang, Shibin Shu, Qi Gu, Zhen Liu, Zezhang Zhu, Yong Qiu, Hongda Bao \\ Spine Surgery, Drum Tower Hospital, Nanjing University Medical School, Nanjing 210008, China \\ Correspondence to: Hongda Bao, MD, PhD. Spine Surgery, Drum Tower Hospital, Nanjing University Medical School, Nanjing 210008, China. \\ Email: baohongda123@gmail.com.
}

\begin{abstract}
Background: Radiographic methods in evaluating the skeletal maturity include Risser sign and TannerWhitehouse score. They are either inappropriate or too complex for a busy clinic setting. The cervical vertebral maturation (CVM) stage is commonly used in orthodontics but has been less acknowledged in studies of spinal growth. The purpose of this study was to evaluate whether the CVM stage could be used as an alternative to Risser sign in determining peak height velocity (PHV).

Methods: This was a two-stage study. Inclusion criteria for stage I study were adolescent female idiopathic scoliosis (AIS) patients, aged between 9-16 years old, who had undergone full spine imaging with clear visibility of the cervical spine. Patients in the stage II study had follow-up through CVM stage 2-4. The correlation between CVM and Risser was analyzed. The spinal growth parameters were measured at each follow-up, and the growth velocity of parameters (PaGVs) was calculated. The PaGVs at CVM stage 2-4 were further compared.

Results: A total of 170 AIS patients were included for stage I study (mean age 12.7 years). The CVM stages were found to correlate strongly with the Risser sign $(\mathrm{r}=0.85, \mathrm{P}<0.01)$. For those patients with Risser stage 0 with closed TC, 71\% were CVM stage 3. Fifty-one patients were included for stage II study. The stature growth velocity averaged $5.4 \mathrm{~cm} /$ year in CVM stage 2 patients and $6.3 \mathrm{~cm} /$ year in CVM stage 3 patients, which was significantly greater growth than that in CVM stage 4 patients $(3.3 \mathrm{~cm} /$ year, both $\mathrm{P}<0.01)$; similarly, the growth velocity of arm span, trunk height, and spinal lengths were also significantly higher in CVM stage 3 patients compared to CVM stage 2 and 4 patients.

Conclusions: The CVM stage could provide an alternative option for the assessment of skeletal maturity of subjects with idiopathic scoliosis. CVM stage 3 may be a new sign of PHV.
\end{abstract}

Keywords: Cervical vertebral maturation; adolescent idiopathic scoliosis; peak height velocity; spinal length growth velocity; skeletal maturity

Submitted Apr 21, 2019. Accepted for publication Oct 28, 2019.

doi: 10.21037/qims.2019.11.07

View this article at: http://dx.doi.org/10.21037/qims.2019.11.07

\section{Introduction}

Adolescent idiopathic scoliosis (AIS) is by far the most common type of scoliosis, affecting children between ages 9 and 18 years (1). The risk of curve progression was found to correlate significantly with the period of rapid skeletal linear growth and in particular, with the time relative to the peak height velocity (PHV) (2), which is defined as the period of the fastest skeletal growth during puberty. The changes in skeletal growth, joint stiffness, flexibility, bone density, and muscle imbalances during PHV are considered to accelerate curve progression (3). Therefore, estimating the PHV plays an important role in determining the bracing strategy and 

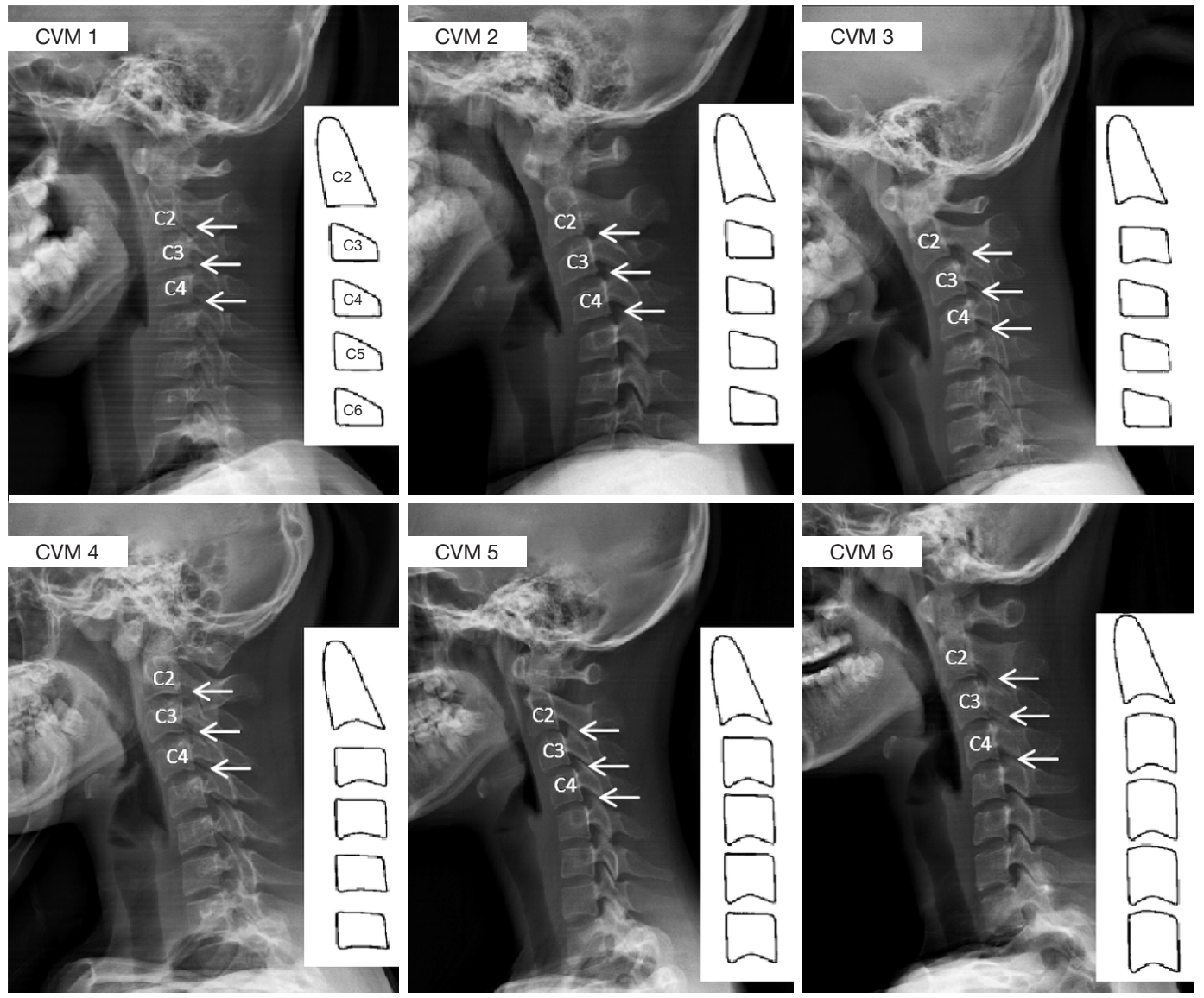

Figure 1 Developmental stages of cervical vertebrae. CVM 1: the lower borders of the second, third, and fourth vertebrae (C2, C3 and C4) are flat, and the bodies of $\mathrm{C} 3$ and $\mathrm{C} 4$ are trapezoidal in shape. CVM 2: only the lower border of $\mathrm{C} 2$ is concave, and the bodies of $\mathrm{C} 3$ and $\mathrm{C} 4$ are trapezoidal. CVM 3: the lower borders of C2 to C3 have concavities, and the bodies of C3 and C4 are either trapezoidal or rectangular horizontal in shape; alternatively, the concavity is present only at the lower border of the $\mathrm{C} 3$ with the bodies of $\mathrm{C} 3$ and $\mathrm{C} 4$ either trapezoidal or rectangular horizontal in shape. CVM 4: the lower borders of $\mathrm{C} 2$ to $\mathrm{C} 4$ have concavities, and the bodies of both $\mathrm{C} 3$ and $\mathrm{C} 4$ are both rectangular horizontal; alternatively, the concavity is present only at the lower border of the $\mathrm{C} 3$ and $\mathrm{C} 4$ with the bodies of $\mathrm{C} 3$ and $\mathrm{C} 4$ rectangular horizontal in shape. CVM 5: the lower borders of $\mathrm{C} 2$ to $\mathrm{C} 4$ have concavities, and at least one or both of the bodies of $\mathrm{C} 3$ and $\mathrm{C} 4$ is square; alternatively, at least the body of either C3 or C4 is square with a lack of concavity at the lower border in either C3 or C4. CVM 6: the lower borders of $\mathrm{C} 2$ to $\mathrm{C} 4$ have concavities, and at least 1 or both of $\mathrm{C} 3$ and $\mathrm{C} 4$ is rectangular vertical (12). CVM, cervical vertebral maturation.

the surgical indications in AIS patients.

There are several assessment methods available to evaluate the PHV, each with their own strengths and weaknesses. The Risser sign debuted in 1958 and gradually became the most commonly used marker for spine surgeons to evaluate skeletal maturation and curve progression (4). The Risser sign appears on the radiograph of the pelvis, which is often studied during the assessment of scoliosis, thereby obviating the need for an additional radiograph (5). However, although the Risser sign is classic, it has several limitations, which include dissymmetry and the long duration of Risser stage 0 . A previous study also showed that it is a poor clinical landmark for noting the beginning of the curve acceleration phase of AIS patients (6). After the Risser sign, came Tanner-Whitehouse (TW) scores (7), digital skeletal age (DSA) scores (2), Sanders simplified skeletal maturity system (8), distal radius and ulna (DRU) stages (9), and the ultramodern thumb ossification composite index (TOCI) (10). In clinical settings, these methods not only need an extra hand radiograph that increases both cost and 
Table 1 Description of the cervical vertebral maturation (CVM) stages (12)

\begin{tabular}{ll}
\hline CVM & Descriptions \\
\hline 1 & $\begin{array}{l}\text { When the lower borders of the second, third and fourth vertebrae(C2,C3 and C4) are flat and the bodies of C3 and C4 are } \\
\text { trapezoidal in shape }\end{array}$ \\
2 & When only the lower border of C2 is concave and the bodies of C3 and C4 are trapezoidal \\
When the lower borders of C2 to C3 have concavities and the bodies of C3 and C4 are either trapezoid or rectangular \\
horizontal in shape. Alternatively, when the concavity is present only at the lower border of the C3 with the bodies of C3 and C4 \\
either trapezoidal or rectangular horizontal in shape \\
When the lower borders of C2 to C4 have concavities and the bodies of both C3 and C4 are both rectangular horizontal. \\
Alternatively, when the concavity is present only at the lower border of the C3 and C4 with the bodies of C3 and C4 rectangular \\
horizontal in shape \\
When the lower borders of C2 to C4 have concavities, and at least one or both of the bodies of C3 and C4 is square. \\
Alternatively, when at least the body of either C3 or C4 is square with a lack of concavity at the lower border in either C3 or C4 \\
When the lower borders of C2 to C4 have concavities, and at least 1 or both of C3 and C4 is rectangular vertical
\end{tabular}

radiation but also are too cumbersome for less experienced surgeons.

In 1975, Lamparski et al. (11) was first to use the cervical vertebrae for skeletal age assessment and was followed by Baccetti et al. (12) who modified the method, changing the name to cervical vertebral maturation (CVM) in 2002. CVM contains 6 different stages that show different geometrical morphology of $\mathrm{C} 2-\mathrm{C} 4$ with the growth of skeletal maturity (Figure 1, Table 1) (12). CVM stage is commonly used in orthodontics but has been neglected in spine research. In previous studies, a significant correlation between CVM and skeletal maturity was identified (13-16). Considering the advantages of radiograph conservation, evaluating spinal growth in AIS patients with CVM may have potential clinical benefit. The main purpose of this study was to determine the reliability of grading with CVM. The secondary objective was to evaluate the relationship between CVM and PHV in AIS patients.

\section{Methods}

\section{Coborts}

This was a two-stage retrospective study approved by the ethics committee of the hospital. In stage I, a retrospective review of 215 patients from a prospectively recruited database between April 2015 and October 2017 was performed. Inclusion criteria for stage I study were (I) female AIS patients, (II) age between 9 and 16 years, (III) who had undergone standardized bracing treatment, (IV) and had full spine images with clear visibility of the cervical spine. The exclusion criteria were (I) patients with previous spinal surgery, (II) any signs of growth abnormalities (such as a lower extremity growth arrest or deficiency), (III) skeletal dysplasia or dwarfism, and (IV) dissymmetry and unclear Risser on the radiograph. For the 215 patients, 30 patients with dissymmetry or unclear Risser, 13 with unavailable radiography, and 2 with abnormalities of skeletal maturation were excluded. Finally, 170 AIS patients were included in stage I of the study.

The stage II study was a longitudinal study; 51 patients who had followed through CVM stage 2 to 4 were identified from the 170 stage I patients and evaluated separately.

\section{Measurements}

For stage I patients, demographic variables were collected when taking radiographs. Full spine radiographs were taken at the initial visit and at each follow-up. The maturity was evaluated using Risser stage and CVM stage. In this study, we defined Risser 0 with the closed triradiate cartilage as Risser 0.5 , and Risser 0 with the opened triradiate cartilage as Risser 0. Risser 0.5 was regarded as a presumed PHV period in this study since a previous study showed that most patients at Risser 0 with closed triradiate cartilage are at PHV. Song et al. (17) proposed that the closure of the triradiate cartilage is closely related to PHV. Nault et al. (6) have proposed that Risser 0 with closed triradiate cartilage indicates a curve acceleration phase when the curve progresses the most rapidly. All radiographs were performed by two spine surgeons and two orthopedic residents. The measurements were performed twice, with a two-week 


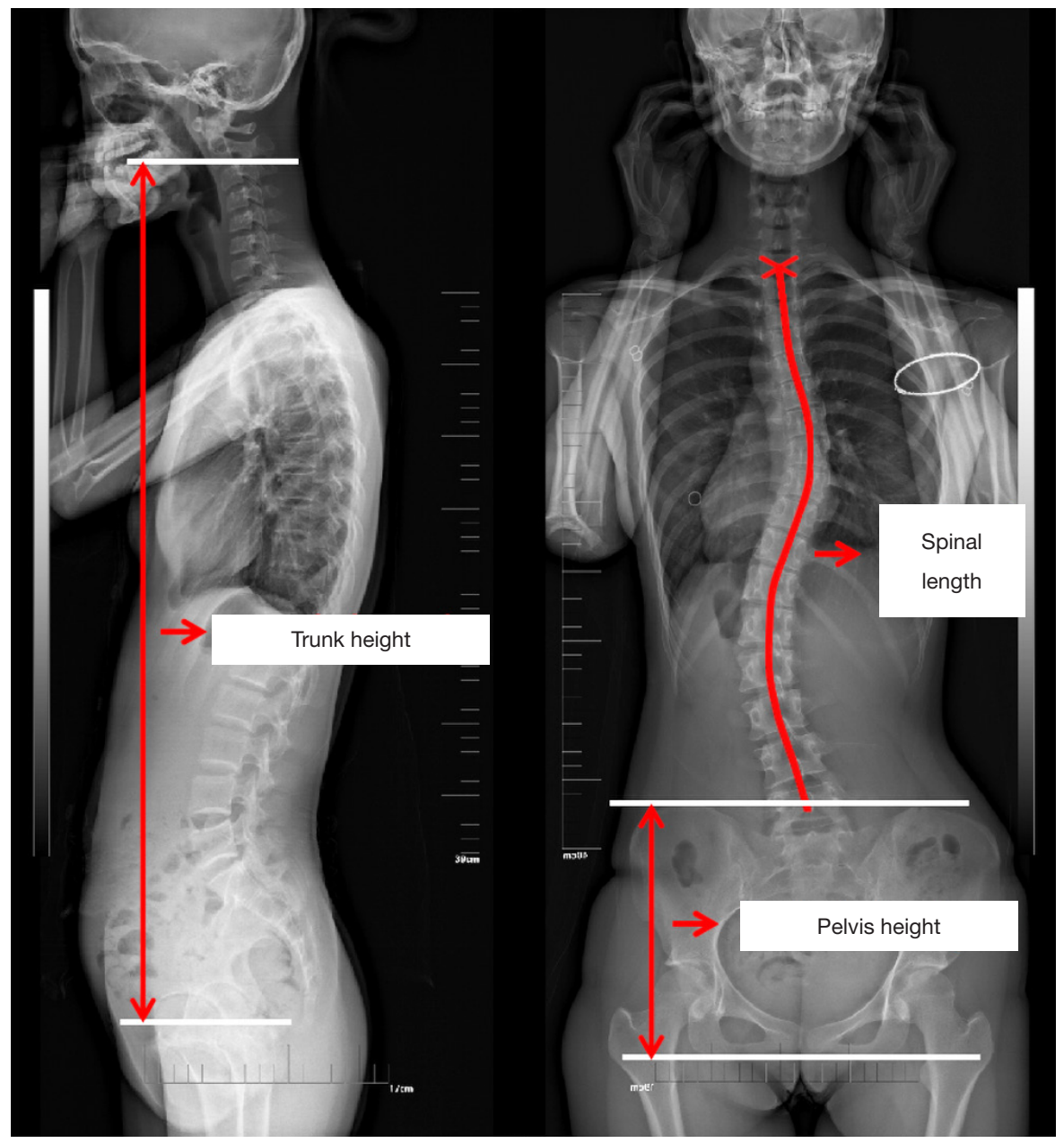

Figure 2 The sagittal trunk height was measured on EOS ${ }^{\mathrm{TM}}$ imaging system (EOS) images as the vertical distance between the horizontal through the midpoint of the $\mathrm{C} 2$ inferior end plate and the horizontal through the midpoint of the femoral head. The coronal spinal length was measured by the line through the midpoints of the superior endplate, diagonal intersection of each vertebra, midpoints of the inferior endplate, and discs from the superior endplate of T1 to the inferior endplate of L5. A horizontal line was passed through the upper margin of the ilium and another through the ischial tuberosity. The vertical distance between these two lines was defined as pelvis height.

interval between each measurement.

For stage II patients, 51 patients of the stage I study had complete follow-up through the CVM stage 2-4 with an interval of 4 to 6 months. Anthropometric measurements were performed at each follow-up, including the stature and the arm span. The sagittal trunk height and coronal spinal length measurements were made on digital images at the picture archiving and communication system (PACS) workstation. The sagittal trunk height was defined as the vertical distance between the horizontal through the midpoint of the $\mathrm{C} 2$ inferior endplate and the horizontal through the midpoint of the femoral head (Figure 2). The intersection of catercorner in each vertebral body was defined as the center of the vertebral body. Total length from radiographs along the line reaching the midpoint of both the superior and inferior endplates from $\mathrm{T} 1$ to L5 and the center of each vertebral body in between were defined as the coronal spinal length $(18,19)$ (Figure 2). A horizontal line was passed through the upper margin of the ilium and another through the ischial tuberosity. The vertical distance between these two lines was defined as the pelvis height (Figure 2).

The calculation of the growth velocity of parameters (PaGVs) was defined as the growth obtained from dividing the parameters increase by the time interval between two 
Table 2 Results of reliability test in the two evaluations in the stage I study

\begin{tabular}{lll}
\hline Measurement & Spine surgeons & Residents \\
\hline First phase & $0.991(0.988-0.993)$ & $0.988(0.984-0.993)$ \\
Second phase (2 weeks later) & $0.996(0.993-0.998)$ & $0.985(0.983-0.990)$ \\
\hline
\end{tabular}

The values are given as the reliability with the $95 \%$ confidence interval in parentheses.

Table 3 The distribution and age of patients at different Risser stages and cervical vertebral maturation (CVM) stages in the stage I study

\begin{tabular}{|c|c|c|}
\hline Indicator & Distribution & Average age (year) \\
\hline \multicolumn{3}{|l|}{ Risser } \\
\hline 0 & 36 & 9.9 \\
\hline 0.5 & 25 & 11.7 \\
\hline 1 & 14 & 12.6 \\
\hline 2 & 27 & 13.2 \\
\hline 3 & 33 & 13.9 \\
\hline 4 & 22 & 14.7 \\
\hline 5 & 13 & 15.2 \\
\hline \multicolumn{3}{|l|}{ CVM } \\
\hline 1 & 15 & 9.6 \\
\hline 2 & 20 & 10.4 \\
\hline 3 & 36 & 11.8 \\
\hline 4 & 45 & 13.0 \\
\hline 5 & 38 & 14.2 \\
\hline 6 & 16 & 15.3 \\
\hline
\end{tabular}

consecutive visits $(2,20)$ :

$$
\mathrm{PaGV}=\left(\text { Parameter }_{\mathrm{n}}-\text { Parameter }_{\mathrm{n}-1}\right) \text { Time interval }_{\mathrm{n}-(\mathrm{n}-1)}
$$

We included the two consecutive visits at the same CVM stage in order to calculate the $\mathrm{PaGV}$ at this CVM stage. The visit, which had a different CVM stage with the last visit was excluded. We then calculated the average $\mathrm{PaGV}$ at the same CVM stages and defined it as the $\mathrm{PaGV}$ at that stage. The ratio of spinal length $v s$. pelvic height (SL $v s$. $\mathrm{PH})$ was defined as the computed result of spinal length divided by the pelvic height. This parameter could show the standard spinal growth using a self-control method.

\section{Statistical analysis}

Data were statistically analyzed with the SPSS Statistics (v 20.0) software packages. The measured values were expressed as mean and standard deviation (SD). Descriptive statistics were performed to analyze patients' demographics. For the intra- and inter-observer reliability analysis, the intraclass correlation coefficient (ICC) was calculated. The Chi-square test was used to compare the percentages of Risser signs and CVM stages in the selected cases. The analysis of variance (ANOVA) was used to compare PaGV at different CVM stages in the stage II study. The Spearman coefficients of correlation were calculated to assess the relationships between Risser signs and CVM stages. $\mathrm{P}<0.05$ was regarded as a statistically significant difference.

\section{Results}

\section{Stage I study}

The mean age was 12.7 (range, 9-16) years. The mean Cobb angle for stage $\mathrm{I}$ at the first visit was $27^{\circ}$ (range, $16^{\circ}-33^{\circ}$ ). All surgeons and residents showed excellent interrater and intrarater agreement for the CVM stages (Table 2).

The distribution of Risser sign in the cohort was as follows: Risser 0 in 36 patients, Risser 0.5 in 25 patients, Risser 1 in 14 patients, Risser 2 in 27 patients, Risser 3 in 33 patients, Risser 4 in 22 patients, and Risser 5 in 13 patients. The distribution of CVM was as follows: CVM stage 1 in 15 patients, CVM stage 2 in 20 patients, CVM stage 3 in 36 patients, CVM stage 4 in 45 patients, CVM stage 5 in 38 patients, and CVM stage 6 in 16 patients. The average age of patients in each Risser stage and CVM stage is shown in Table 3. The CVM stages were found to correlate strongly with Risser sign $(\mathrm{r}=0.85, \mathrm{P}<0.01)$.

The percentage of CVM stages in Risser stages is shown in Figure 3. In the period of Risser 0, there are 39\% of patients at CVM stage 1,39\% of patients at CVM stage 2, while the remaining $22 \%$ are at CVM stage 3 . In the period of Risser 0.5, which indicates the stage of PHV, there are $16 \%$ of patients at CVM stage $2,71 \%$ of patients at CVM stage 3, while the remaining $13 \%$ are at CVM stage 3 . In the total period of Risser sign 0.5 and 1 , there are only $9 \%$ and $3 \%$ of patients at CVM stage 2 and CVM stage 

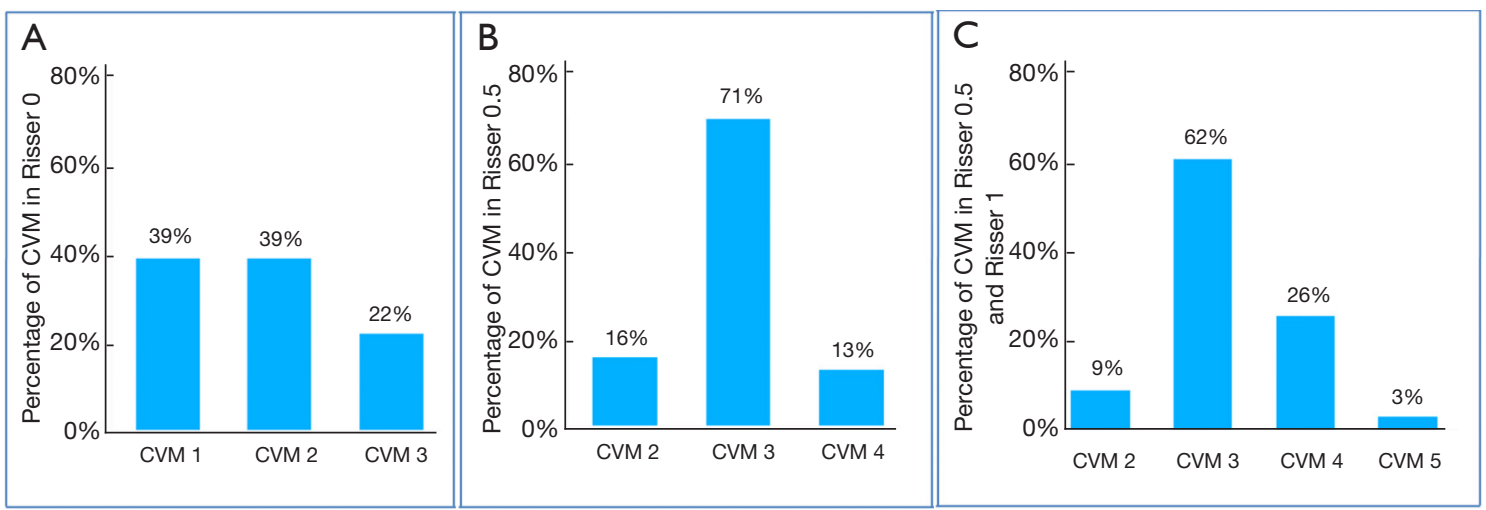

Figure 3 Percentage of CVM stages in Risser stages in the stage I study. The percentage of CVM stage 1-3 in Risser 0 (A); the percentage of CVM stage 2-4 in Risser 0.5 (B); the percentage of CVM stage 2-5 in Risser 0.5 and Risser 1 (C) (Risser 0.5 is Risser 0 with closed triradiate cartilage). CVM, cervical vertebral maturation.

Table 4 The parameters at different CVM stages in the stage II study

\begin{tabular}{lccccc}
\hline CVM & S $(\mathrm{cm})$ & AS $(\mathrm{cm})$ & TH $(\mathrm{cm})$ & SL $(\mathrm{cm})$ \\
\hline 1 & $143.6(131.5-152.6)$ & $144.8(132.3-153.2)$ & $55.2(51.6-58.7)$ & $44.3(42.6-47.7)$ & 2.40 \\
2 & $146.2(134.6-155.2)$ & $147.3(134.8-155.9)$ & $56.3(52.4-59.3)$ & $45.1(42.8-48.1)$ & 2.38 \\
3 & $154.6(144.0-162.2)$ & $155.7(145.1-164.4)$ & $59.5(54.3-60.1)$ & $47.1(43.7-50.5)$ & 2.34 \\
4 & $158.7(148.9-167.7)$ & $159.7(149.3-167.2)$ & $61.1(57.6-63.8)$ & $48.4(44.9-51.0)$ & 2.34 \\
5 & $161.0(152.3-170.9)$ & $162.1(152.2-171.6)$ & $61.3(57.9-64.6)$ & $48.9(45.4-52.6)$ & 2.34 \\
6 & $161.2(152.3-172.0)$ & $162.8(152.3-172.7)$ & $62.0(58.7-65.0)$ & $49.1(46.7-52.7)$ & 2.31 \\
\hline
\end{tabular}

CVM, cervical vertebral maturation; S, stature; AS, arm span; TH, trunk height; SL, spinal length; PH, pelvis height.

Table 5 The parameters growth velocity at different CVM stages in the stage II study

\begin{tabular}{lcccc}
\hline CVM & S (cm/year) & AS (cm/year) & TH (cm/year) & SL (cm/year) \\
\hline 2 & 5.4 & 5.5 & 2.15 & 1.45 \\
3 & 6.3 & 6.2 & 2.40 & 1.65 \\
4 & 3.3 & 3.2 & 1.06 & 0.97 \\
P value & $<0.001$ & $<0.001$ & 0.001 & 0.004 \\
\hline
\end{tabular}

CVM, cervical vertebral maturation; S, stature; AS, arm span; $\mathrm{TH}$, trunk height; SL, spinal length; $\mathrm{PH}$, pelvis height.

5 , respectively, and $62 \%$ of patients at CVM stage 3 . The other $26 \%$ are at CVM stage 4 .

\section{Stage II study}

The stature, arm span, trunk height, and spinal lengths of patients at different CVM stages are shown in Table 4. The growth velocity of parameters (PaGVs) in different CVM stages is shown in Table 5. The stature growth velocity averaged $5.4 \mathrm{~cm} /$ year in CVM stage 2 and $6.3 \mathrm{~cm} /$ year in CVM stage 3, which is significantly larger than that in CVM stage $4(3.3 \mathrm{~cm} /$ year, both $\mathrm{P}<0.001)$; similarly, the arm span growth velocity was also significantly higher in CVM stage 3 than in CVM stage 2 and CVM stage 4 (6.2 vs. 5.5 and $3.2 \mathrm{~cm} /$ year, both $\mathrm{P}<0.001)$. The trunk height growth velocity averaged $2.15 \mathrm{~cm} /$ year in CVM stage 2 and $2.40 \mathrm{~cm} /$ year in CVM stage 3, which are both significantly larger than that in CVM stage $4(1.06 \mathrm{~cm} /$ year, both $\mathrm{P}<0.001)$. The spinal length growth velocity averaged $1.45 \mathrm{~cm} /$ year in CVM stage 2 and $1.65 \mathrm{~cm} /$ year in CVM stage 3, which are both significantly larger than that in CVM stage $4(0.97 \mathrm{~cm} /$ year, both $\mathrm{P}<0.001)$. The ratio of spinal length $v s$. pelvis height showed no significant difference (Table 4), indicating a normal trend of spinal growth in all subjects. 


\section{Discussion}

An ideal maturity indicator should not only be easy to measure, but it should also be convenient for patients, have little diurnal variation, and correlate well with the timing of curve progression. The results of this study revealed that CVM stage showed strong correlation with Risser sign. Patients at CVM stage 3 made up $71 \%$ of those subjects at Risser 0 with closed triradiate cartilage, indicating that patients at CVM stage 3 are most likely at PHV, which was further validated by the calculation of the PaGVs.

In earlier generations, the cervical vertebrae were used to assess skeletal maturity (11), but no specific grades were defined. Baccetti et al. (21) modified the assessment of skeletal maturity using the cervical vertebrae and presented a simple method of determining the skeletal age using six developmental stages of the cervical vertebrae (C2-4), with each developmental stage being a biological growth indicator. Adding on to the proven usefulness of this method in orthodontic research, our study extended CVM stages to the evaluation of spinal growth. CVM staging should be one of the methods used in evaluating the spinal maturity in scoliosis patients since the cervical vertebrae is an internal part of the spine. The changing of cervical vertebrae could represent the exact stage of thoracic and lumbar spinal growth, and should theoretically be better than the prediction ability of metacarpals or the radius and ulna. The morphology of a cervical vertebra is straightforward, and the concave of each vertebra is obvious and clearly visible (Figure 4).

It should be mentioned that the CVM staging still entails several limitations, including the inconsistent changes in skeletal development during the growth period and nonquantified measurements of bone mass (22). To improve the CVM stage, Chen et al. (23) used a quantitative analytical method from the sagittal view and presented an objective indicator replacing the lateral cephalometric radiography. Yang et al. (24) also performed quantitative shape analysis, which enhanced the ability to explain bone maturation as opposed to relying only on chronological age. In our study, we used the classic CVM grades instead of quantified evaluation since we were seeking an easily understood and convenient method for an outpatient clinic, and the quantified evaluation should be further analyzed in the future.

In our study, we found the percentages of patients in CVM stage 1 and CVM stage 2 in Risser 0 with open triradiate cartilage were both $39 \%$, indicating that CVM stage 1 and CVM stage 2 can represent the Risser 0 with open triradiate cartilage. CVM stage 1 or CVM stage 2 may predicate the potential acceleration of curve progression. Previous studies showed that most patients with Risser 0 with closed triradiate cartilage are at PHV $(6,17)$. In our study, $71 \%$ of CVM 3 patients were Risser 0 with closed triradiate cartilage. Therefore, CVM 3 may be an indicator of PHV. To validate this hypothesis, this study also included 51 patients with complete follow-up through the brace treatment and calculated the PaGVs for different CVM stages. The results revealed that the growth velocity of stature, arm span, trunk height, and spinal lengths was all significantly higher in CVM stage 3, which supports the results indicating that CVM stage 3 can be an indicator of PHV.

The risk of curve progression is primarily correlated to periods of rapid spinal growth. Stature is a useful representation of spinal growth. Despite being helpful as a first indication for the curve acceleration phase, the predictive values of stature velocity were partly downgraded by the existence of the distal-to-proximal growth gradient in adolescents. The peak growth of distal body parts, for example, foot length or lower limb length was found to precede the peak growth of more proximal body parts including the spine (25). This growth gradient may introduce deviations when we use the height growth velocity as the first-line predictor of curve progression. The risk of curve progression incorporates the lower limbs and pelvis; it may not be as sensitive for monitoring spine growth in managing deformity as the trunk height, which eliminates the contribution of the lower limbs. In this study, with our use of stature combined with arm span for measuring growth velocity, the growth data may be more representative of long bone growth rather than spine growth. Arm span is useful in patients with scoliosis because curve deterioration may cause a reduction in overall height and thus mask the actual spine growth achieved (26). Therefore, we employed the measurement of spinal length and trunk height for better evaluation of growth velocity. As we expected, the growth velocity of all parameters was higher in CVM stage 3 (Table 5), demonstrating the potential clinical use of CVM grade.

It has been well documented that the growth and curve progression in AIS is closely interrelated. Sanders et al. (2) proved that the timing relative to $\mathrm{PHV}$, which significantly correlated with the curve progression phase could be predicted. An increase of stature more than $4 \mathrm{~cm} /$ year with curve progression more than $25^{\circ}$ was significantly associated with an increase in the angle velocity (27). Therefore, an efficient method to evaluate maturity status is constantly 

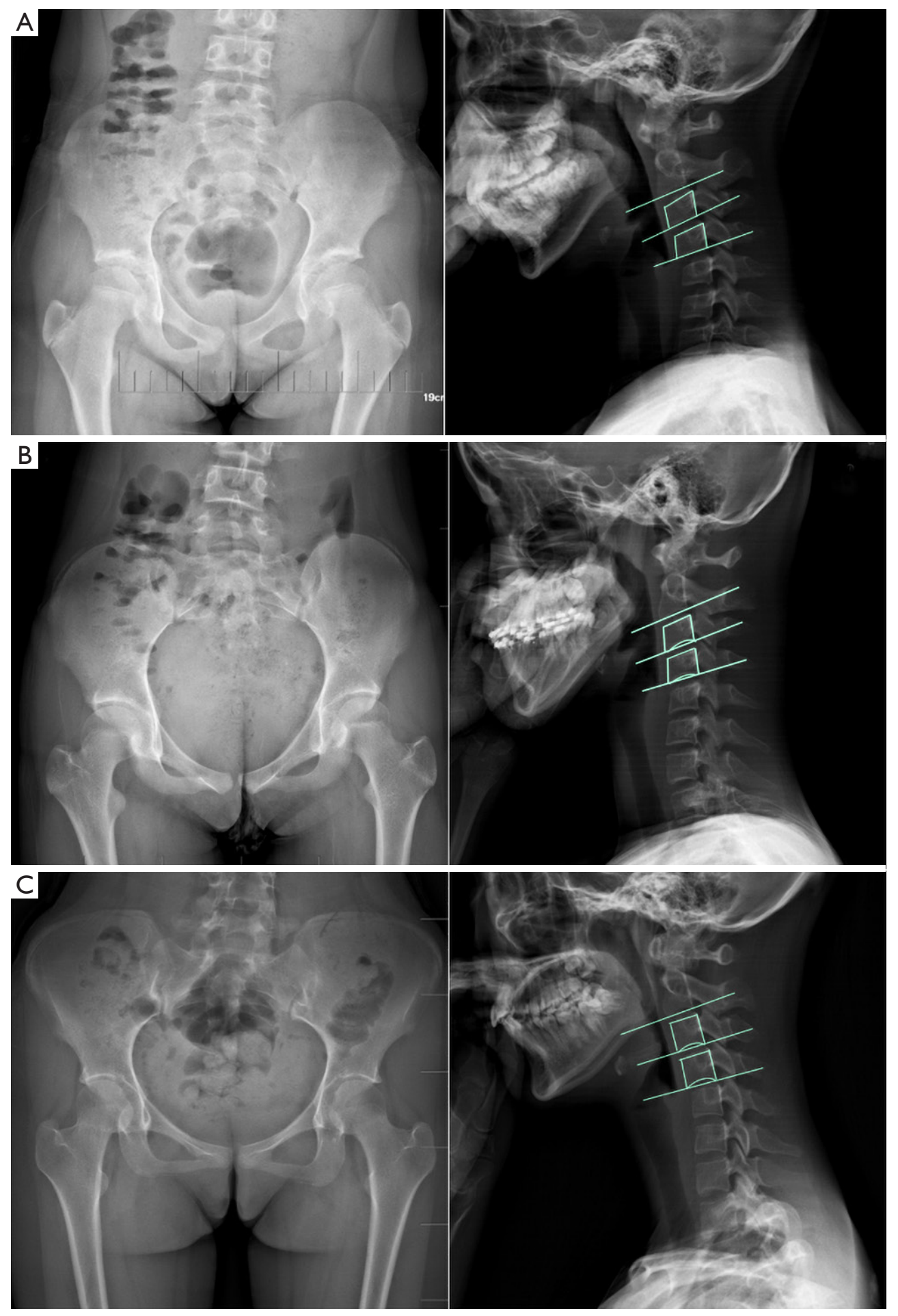

Figure 4 Three cases. A 10.6-year-old girl at Risser 0 and CVM stage 2 (A); a 13.3-year-old girl at Risser 2 and CVM stage 4 (B); a 14.5-year-old girl at Risser 4 and CVM stage 5 (C). CVM, cervical vertebral maturation. 
being sought by surgeons to determine the treatment strategy. Bracing before the PHV has been shown to prevent curve progression (28-30). Bracing strategy, including the initiation of bracing, timing of weaning, and the hours of brace wear, should be determined based on maturity status and growth potential. Therefore, the CVM stages could be potentially used in guiding bracing treatment and should be further investigated.

There are several limitations to this study. First, the patients in the stage 1 study only had one-time followup. In further study, we will particularly select patients with more follow-ups through whole skeletal maturity. Second, the study only analyzed the correlation between CVM stages and Risser stage. Although Risser stage is the most commonly used method, it is not considered to be the most accurate for evaluating skeletal maturity. Using TOCI or DSA would have been appropriate, but the hand radiographs were incomplete in long-term follow-up. Third, the patients were all treated with brace. The time interval between two consecutive visits was 4 to 6 months, which was too long to observe the growth. The brace may also influence the growth of each patient.

\section{Conclusions}

The new CVM stage could provide an alternative option for the assessment of skeletal maturity of subjects with idiopathic scoliosis. CVM3 may be a new sign of PHV. The index needs to be verified in further multicenter studies with different ethnic groups.

\section{Acknowledgments}

Funding: This work received funding from the Natural Science Foundation of Youth Fund Projects of Jiangsu Province (BK20180122).

\section{Footnote}

Conflicts of Interest: The authors have no conflicts of interest to declare.

Ethical Statement: This was a two-stage retrospective study approved by the ethics committee of the hospital.

\section{References}

1. Ylikoski M. Growth and progression of adolescent idiopathic scoliosis in girls. J Pediatr Orthop B

2005;14:320-4.

2. Sanders, James O. Maturity Indicators in Spinal Deformity. J Bone Joint Surg 2007;89:14.

3. Calvo IJ. Observations on the growth of the female adolescent spine and its relation to scoliosis. Clin Orthop 1957;10:40-7.

4. Risser JC. The Iliac apophysis; an invaluable sign in the management of scoliosis. Clin Orthop 1958;11:111-9.

5. Little DG, Sussman MD. The Risser sign: a critical analysis. J Pediatr Orthop 1994;14:569-75.

6. Nault ML, Parent S, Phan P, Roy-Beaudry M, Labelle H, Rivard M. A Modified Risser Grading System Predicts the Curve Acceleration Phase of Female Adolescent Idiopathic Scoliosis. J Bone Joint Surg Am 2010;92:1073-81.

7. Tanner JM, Whitehouse RH, Cameron N, Marshall WA, Healy MJ, Goldstein H. Assessment of skeletal maturity and prediction of adult height (TW2 method). London: Academic Press; 1983.

8. Sanders, James O. Predicting Scoliosis Progression from Skeletal Maturity: A Simplified Classification During Adolescence. J Bone Joint Surg Am 2008;90:540-53.

9. Luk KD, Saw LB, Grozman S, Cheung KM, Samartzis D. Assessment of skeletal maturity in scoliosis patients to determine clinical management: a new classification scheme using distal radius and ulna radiographs. Spine J 2014;14:315-25.

10. Hung ALH, Chau WW, Shi B, Chow SK, Yu FYP, Lam TP, Ng BKW, Qiu Y, Cheng JCY. Thumb Ossification Composite Index (TOCI) for Predicting Peripubertal Skeletal Maturity and Peak Height Velocity in Idiopathic Scoliosis. J Bone Joint Surg Am 2017;99:1438-46.

11. Lamparski DG. Skeletal age assessment utilizing cervical vertebrae. Am J Orthod 1975;67:458-9.

12. Baccetti T, Franchi L, McNamara JA Jr. An improved version of the cervical vertebral maturation (CVM) method for the assessment of mandibular growth. Angle Orthod 2002;72:316-23.

13. Franchi L, Baccetti T, McNamara JA Jr. Mandibular growth as related to cervical vertebral maturation and body height. Am J Orthod Dentofacial Orthop 2000;118:335-40.

14. Hassel B, Farman AG. Skeletal maturation evaluation using cervical vertebrae. Am J Orthod Dentofacial Orthop 1995;107:58-66.

15. O'Reilly MT, Yanniello GJ. Mandibular growth changes and maturation of cervical vertebrae--a longitudinal cephalometric study. Angle Orthod 1988;58:179-84. 
16. Beit P, Peltomäki T, Schätzle M, Signorelli L, Patcas R. Evaluating the agreement of skeletal age assessment based on hand-wrist and cervical vertebrae radiography. Am J Orthod Dentofacial Orthop 2013;144:838-47.

17. Song KM, Little DG. Peak height velocity as a maturity indicator for males with idiopathic scoliosis. J Pediatr Orthop 2000;20:286-8.

18. Wever DJ, Tonseth KA, Veldhuizen AG. Curve progression and spinal growth in brace treated idiopathic scoliosis. Clin Orthop Relat Res 2000;(377):169-79.

19. Cheung J, Veldhuizen AG, Halberts JP, Sluiter WJ, Van Horn JR. Geometric and electromyographic assessments in the evaluation of curve progression in idiopathic scoliosis. Spine (Phila Pa 1976) 2006;31:322-9.

20. Escalada F, Marco E, Duarte E, Ma Muniesa J, Boza R, Tejero M, Cáceres E. Assessment of angle velocity in girls with adolescent idiopathic scoliosis. Scoliosis 2009;4:20.

21. Baccetti T, Franchi L, Mcnamara JA Jr. The Cervical Vertebral Maturation (CVM) Method for the Assessment of Optimal Treatment Timing in Dentofacial Orthopedics. Semin Orthod 2005;11:119-29.

22. Gabriel DB, Southard KA, Qian F, Marshall SD, Franciscus RG, Southard TE. Cervical vertebrae maturation method: Poor reproducibility. Am J Orthod Dentofacial Orthop 2009;136:478.e1-7; discussion 478-80.

23. Chen L, Liu J, Xu T, Long X, Lin J. Quantitative skeletal evaluation based on cervical vertebral maturation: a longitudinal study of adolescents with normal occlusion. Int J Oral Maxillofac Surg 2010;39:653-9.

Cite this article as: Zhang Y, Shu S, Gu Q, Liu Z, Zhu Z, Qiu Y, Bao H. Cervical vertebral maturation (CVM) stage as a supplementary indicator for the assessment of peak height velocity (PHV) in adolescent idiopathic scoliosis (AIS). Quant Imaging Med Surg 2020;10(1):96-105. doi: 10.21037/ qims.2019.11.07
24. Yang YM, Lee J, Kim YI, Cho BH, Park SB. Axial cervical vertebrae-based multivariate regression model for the estimation of skeletal-maturation status. Orthod Craniofac Res 2014;17:187-96.

25. Busscher I, Gerver WJ, Kingma I, Wapstra FH, Verkerke GJ, Veldhuizen AG. The growth of different body length dimensions is not predictive for the peak growth velocity of sitting height in the individual child. Eur Spine J 2011;20:791-7.

26. Cheung JP, Cheung PW, Samartzis D, Cheung KM, Luk $\mathrm{KD}$. The use of the distal radius and ulna classification for the prediction of growth: peak growth spurt and growth cessation. Bone Joint J 2016;98-B:1689-96.

27. Yrjönen T, Ylikoski M. Effect of growth velocity on the progression of adolescent idiopathic scoliosis in boys. J Pediatr Orthop B 2006;15:311-5.

28. Weinstein SL, Dolan LA, Wright JG, Dobbs MB. Effects of Bracing in Adolescents with Idiopathic Scoliosis. N Engl J Med 2013;369:1512-21.

29. Sanders JO, Newton PO, Browne RH, Herring AJ. Bracing in adolescent idiopathic scoliosis, surrogate outcomes, and the number needed to treat. J Pediatr Orthop 2012;32 Suppl 2:S153-7.

30. Sitoula P, Verma K, Holmes L Jr, Gabos PG, Sanders JO, Yorgova P, Neiss G, Rogers K, Shah SA. Prediction of Curve Progression in Idiopathic Scoliosis: Validation of the Sanders Skeletal Maturity Staging System. Spine (Phila Pa 1976) 2015;40:1006-13. 